\title{
LASER-BASED ADAPTIVE CRUISE CONTROL FOR INTELLIGENT VEHICLES
}

\author{
Miguel Ángel Sotelo, IEEE Member, David Fernández \\ Department of Electronics, University of Alcalá, Alcalá de Henares, Madrid, Spain \\ Email:michael@depeca.uah.es,llorca@depeca.uah.es \\ Eugenio Naranjo, Carlos González, IEEE member, Ricardo García, Teresa de Pedro, Jesús Reviejo \\ Department of Industrial Computer Science, Industrial Automation Institute, CSIC, Arganda del Rey, Madrid, Spain \\ Email: jnaranjo@iai.csic.es,cgonzalez@iai.csic.es,ricardo@iai.csic.es,tere@iai.csic.es,jreviejo@iai.csic.es
}

\begin{abstract}
Keywords: Intelligent Transportation Systems; Laser-based Adaptive Cruise Control; Fuzzy Logic; Vehicle Automation

Abstract: Vehicle and highway automation is believed to reduce the risk of accident, improve safety, increase capacity, reduce fuel consumption and enchance overall comfort and performance for drivers. One of the most important research topics in the field of Intelligent Transportation Systems (ITS) is Adaptive Cruise Control (ACC), aiming at adapting the vehicle speed to a predefined value while keeping a safe gap with regard to potential obstacles. For this purpose, a laserscanner system provides the distance between the ego vehicle and the preceding vehicle on the road. The complete system can be understood as a Laser-based ACC controller, based on Fuzzy Logic, which assists the vehicle velocity control offering driving strategies and actuation over the throttle of a car. This controller is embedded in an automatic driving system installed in two testbed mass-produced cars operating in a real environment. The results obtained in these experiments show a good performance of the Laser-based gap controller, which is adaptable to all speeds and safe gap selections.
\end{abstract}

\section{INTRODUCTION}

Recent studies estimate that some 5.5 million European Union accidents resulted in 42200 fatalities in 1998. The United States that same year claimed another 42000 lives, and 9000 more were lost in Japan. Overall, the cost to these nations totalled some $€ 682$ billion, as described in (Marsh, 2003). As a result, a lot of money has been spent in order to make roads safer. One of the applications of ITS is to provide assistance to the control of some vehicle elements, like the throttle pedal and consequently, the speed-control assistance. A Cruise Control (CC) system is a common application of these techniques. It consists of maintaining the vehicle speed at a user (driver) pre-set speed. These kind of systems are already mass installed in top of the line-end vehicles. A second and more sophisticated step in the development of the speed assistances is Adaptive Cruise Control (ACC) (Crosse, 2000). ACC is similar to conventional cruise control in that it keeps the vehicle pre-set speed. However, unlike conventional cruise control, this new system can automatically adjust speed in order to maintain a proper headway distance (gap) between vehicles in the same lane (STARDUST, 2002). In the current work this is achieved through a laserscanner headway sensor, a PC, and a fuzzylogic speed controller. This paper addresses the integration of a lasersacnner system, mechatronics, and fuzzy control techniques in order to get robotic aids to driving cars. The present application includes a car computer throttle control powered by a fuzzy logic controller, with the capability of performing a Laser-based Adaptive Cruise Control either in an unmanned/manual driving. The work described in this paper was done at the Instituto de Automática Industrial (IAI), a part of the Spanish Council for Scientific Research (CSIC), in collaboration with the Department of Electronics of the University of Alcalá. The experiments were made in a private test circuit located at the Instituto de Automática Industrial, using two automated mass-produced vehicles (Citroën Berlingo) as depicted in figure 1. 


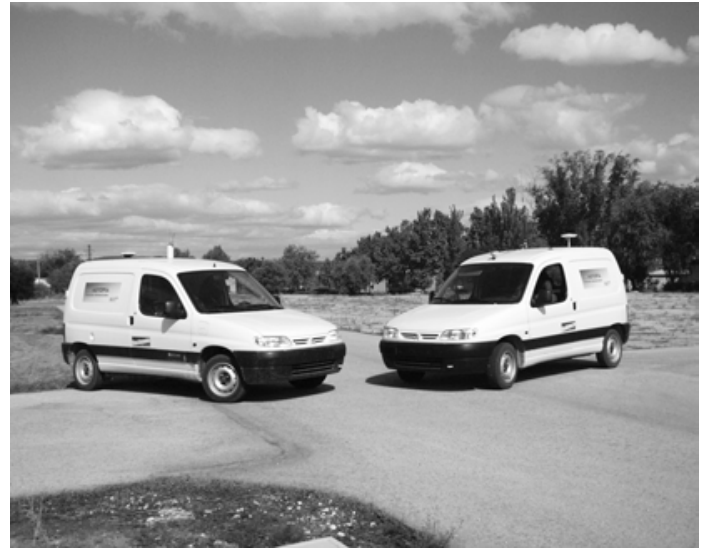

Figure 1: Citroën Berlingo commercial prototypes.

\section{LASER-BASED OBSTACLE DETECTION}

A laserscanner (SICK LMS) is mounted onboard the vehicle in order to provide reliable obstacle detection in a range that can reach up to $90 \mathrm{~m}$, depending on weather conditions. Using the information obtained from the laserscanner, a map of the environment around the vehicle can be constructed and thus, vehicle velocity can be modified so as to track the preceding vehicle in an adaptive cruise control manner (ACC) or to produce an emergency stop in case of inminent collision. Scan data provided by the laserscanner are converted to $x-y$ coordinates with respect to the vehicle frontal part. Other vehicles can then be easily detected by selecting the appropriate ROI in each situation. To gain a better understanding on how the real system works in real circumstances an example is presented in which the ego-vehicle is driving along a straight section of road, $62 \mathrm{~m}$ behind the preceding vehicle, as depicted in figure $2 \mathrm{a}$. The angular resolution of the SICK LMS was set to $0.25^{\mathrm{B}}$ in this case, as long as ACC is the desired feature. Distances measured by the laserscanner at different angles are shown in figure $2 b$, while the $x-y$ coordinates of the detected obstacles are depicted in figure $2 \mathrm{c}$.

\section{LASER-BASED ACC}

The Laser-based Adaptive Cruise Control (ACC) is based on a fuzzy Adaptive Cruise Control System. Although a detailed description of the fuzzy ACC can be found in (Naranjo et al, 2003), a brief summary is provided.

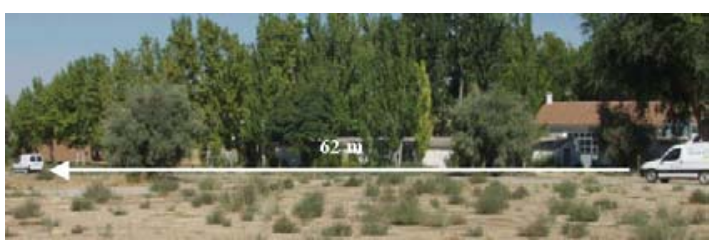

a)

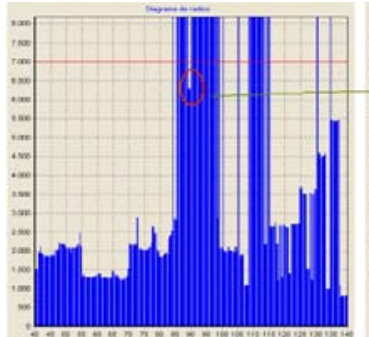

b)

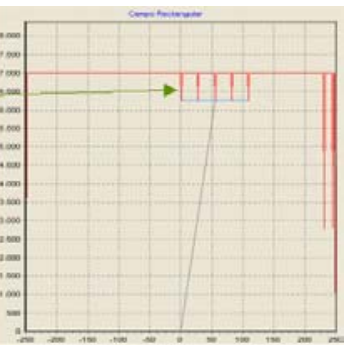

c)
Figure 2: Vehicle detected on the same lane. a) Scene picture, b) Distances measured by the laserscanner, c) $x-y$ coordinates of detected obstacles.

The ACC control system is based on the fuzzy CC described in (Naranjo et al, 2003), with its objective being to maintain a safe gap with the vehicle ahead in the same lane of the road. Two new input fuzzy variables, a new rule and two rule modifications were added to the controller in order to perform the ACC. The output is the same as that in the CC controller: the actuation over the throttle pedal. The pursuer car will be the automatically driven vehicle that adapts its speed to the preceding one. At this point, we shall define the new input variables:

Time_Gap_current: it is the time headway, the time it takes the pursuer vehicle to reach the point where the pursued vehicle is at present speed, in seconds. The mathematical representation is (1).

Time_Gap current $_{1}=\frac{x_{\text {Pursued }}-x_{\text {Pursuer }}}{v_{\text {Pursuer }}}$

where $\mathrm{x}_{\text {Pursued }}$ and $\mathrm{x}_{\text {Pursuer }}$ are the $\mathrm{x}$ coordinate of the pursued and the pursuer cars over the reference line, in meters, and $v_{\text {Pursuer }}$ is the speed of the pursuer car in meters per second.

Time Gap target: it is the time-headway set by the user to be maintained by the ACC system from the preceding vehicle. In commercial vehicles it should be between 1 and 2 seconds.

Derivative of Time Gap: is the variation of the Time-Gap_current with time. Its mission is to smooth the actuation in the same way that the Acceleration smoothes the Speed_Error. The 
equation used to calculate this variable for the control iteration $i$ is (2).

$d_{-}$Time_Gap ${ }_{i}=\frac{\text { Time_Gap }_{i}-\text { Time }_{-} G a p_{i-4}}{4 \Delta t}$

Time_Gap_Error: it represents the time-gap error, the difference between the user-defined target time-gap and the current time-gap. Then, the input value for the gap fuzzy controller is represented in (3) and measured in seconds.

Time_Gap_Error $=T i m e \_G a p_{\text {current }}-$ Time_Gap target $_{-}$

We added a new rule and we also modified two $\mathrm{CC}$ previous rules. The new set is as follows:

IF Speed_Error MORE THAN null THEN Accelerator up

IF Speed_Error LESS THAN null AND Time Gap Error MORE THAN near THEN Accelerator down

IF Acceleration MORE THAN null THEN Accelerator up

IF Acceleration LESS THAN null AND Time_Gap_Error far THEN Accelerator down

IF Time_Gap_Error near AND $d$ _Time_Gap negative THEN Accelerator up

The aim of these rules is to maintain the Cruise Control and to keep a safe distance. To do this, the gap control only actuates when the pursuer car is near the pursued one and it inhibits itself in other cases, the control thus becoming the classical CC. Let us see in detail the modified acceleration rules.

IF Speed_Error LESS THAN null AND Time_Gap_Error MORE THAN near THEN Accelerator down

The throttle signal decreases when the pursuer car is near the pursued one so it will never accelerate enough to crash with the other car.

IF Acceleration LESS THAN null AND Time_Gap_Error far THEN Accelerator down

This rule allows stepping on the throttle only when the pursuer car is far from the pursued one.

IF Time_Gap_Error near AND $d$ _Time_Gap negative THEN Accelerator up

With this rule, the control steps off the throttle when the safe distance is near. The stabilization of the system is the reason for the inclusion of the derivative in this rule.

\section{EXPERIMENTAL RESULTS}

After installing the above defined controller in the testbed cars, some experiments were made in order to demonstrate its performance. These tests were done at the CSIC's Instituto de Automática Industrial in Arganda del Rey, Madrid. The experiment set consists of the combination of laserbased safe gap maintenance and stop-and-go capacity. Two testbed cars were used to make the controller tests. Both of them are equipped with a computer, an RTK-GPS receptor and RadioEthernet, but only the pursuer has an onboard laserbased ACC. The pursued is manually driven in order to simulate real conditions in which the reactions of the car ahead are unpredictable. The $\mathrm{CC}$ will be in effect when the car is alone on the road. During the 156 seconds of this experiment, the pursued car runs at some variable speed between 0 , when the car is stopped, and $30 \mathrm{Km} / \mathrm{h}$. The target speed of the pursuer car is always higher than the pursued one so, the cruise control will only maintain this speed when the pursuer is farther than the pre-selected safe gap. The experiment was made in a circuit with a straight lane with $2 \mathrm{Km}$ of length, oriented from West to East, yielding the results depicted in figure 3 .
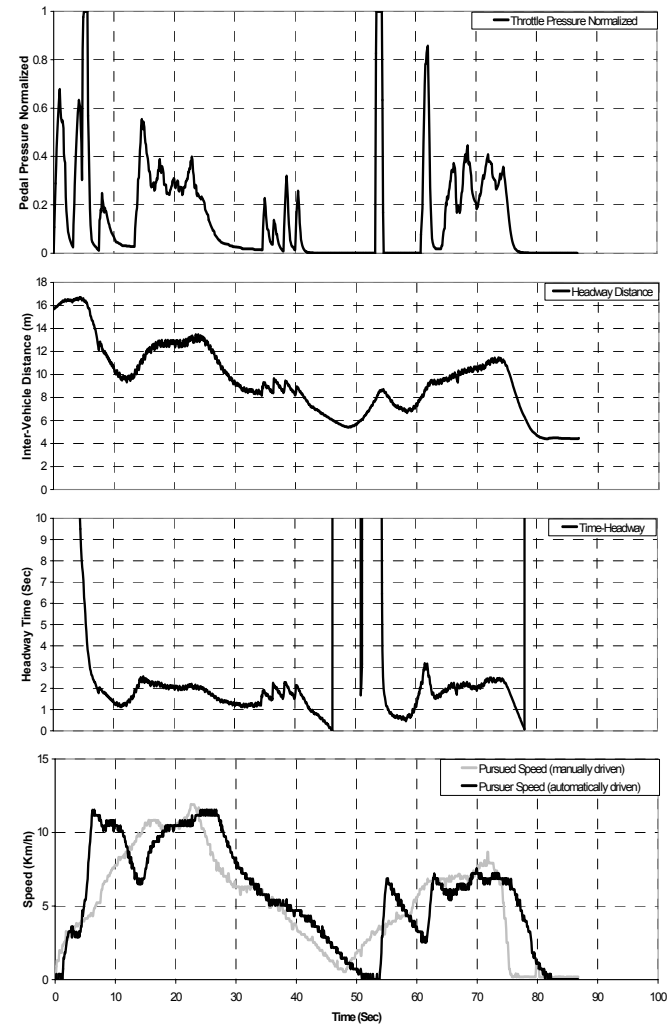

Figure 3: Fuzzy ACC performance. 
We have also pre-set a minimum safe headway gap in the pursuer car of 2 seconds. The reason for selecting this value is that 2 seconds are enough to fulfil the safety requirements in a limited environment such as our circuit. The bottom graphic in figure 3 shows the real speed of both cars for the duration of the experiment. The third graphic is the real-time headway time-gap between these testbed cars. The second represents the inter-vehicle distance, in meters, including the length of the pursued car $(4 \mathrm{~m})$. The top graphic shows the pressure on the throttle of the pursued car, meaning 0 foot quite off the pedal and 1 throttle fully pressed. At the beginning of the experiment both cars are stopped and separated by about 50 meters. The driver of the pursued car starts slowly while the automatic driver of the pursued car sets the target speed at $8 \mathrm{Km} / \mathrm{h}$. The time gap is initially very high, because the speeds are too low, so as the pursuer car speed increases, the gap reduces. After the first 16 seconds, the pursuer car gets to its target speed of 20 $\mathrm{Km} / \mathrm{h}$. Then, the gap reduces drastically until it becomes about 2 seconds. At 40 seconds from the beginning, the pursued car stops. In this case, the pursuer car approaches the other car until the gap is about 2 meters (6 in the graphic), when it stops too (STOP). The reason for this change of units is that when the pursuer speed tends to zero, the time-gap tends to infinity and in this case it is not useful for control, because the cars would crash. It can be seen in the gap graphic around the 40th second. The distance between the cars is never less than 2 meters. In order to improve the safety at these low speeds it is recommended to increase the minimum safe gap to 3 or 4 seconds.

\section{CONCLUSIONS}

The alliance of laser technology, fuzzy logic, and Global Navigation Satellite Systems (GNSS) can generate powerful controllers for automatic driving applications. The combination of ACC+Stop\&Go is a good solution in order to achieve safer driving, from high workload roads to traffic jams. A SICK LMS 221 is the key element to provide obstacle detection for active safety. By selecting a configurable Region of Interest (ROI), the detection ability of the laser system can be adapted to quite different driving situations such as Adaptive Cruise Control (tracking of a preceding vehicle on the same lane), overtaking manoeuvres, and intersection navigation (giving way to other vehicles before traversing the intersection). This makes the system a very versatile one and allows to use it either on highways or on urban scenarios. In our experiments, one of the testbed vehicles is manually driven while the second vehicle is autonomously driven using the laser-based ACC system described in this work. The real application of this kind of technology can be grouped in the field of intelligent driving aids.

\section{ACKNOWLEDGEMENT}

This work has been granted by several Spanish Foundations, being the last ones: Ministery of Science CICYT DPI2002-04064-05-04 and Ministery of Fomento (Transports).

\section{REFERENCES}

Jones, W. D., 2001. Keeping Cars from Crashing. In IEEE Spectrum, pp. $40-45$.

Crosse, J., 2000. Tomorrow's World, Automotive World, pp. $46-48$.

STARDUST, 2002. Scenarios and Evaluation Framework for City Case Studies. European Comission Fifth Framework Programme Energy, Environment and Sustainable Development Programme Key Action 4: City of Tomorrow and Cultural Heritage, Deliverable 2, 3 .

Ioannou, P. A. and Chien, C. C., 1993. Autonomous Intelligent Cruise Control. IEEE Transactions on Vehicular Technology, pp. 657-672, volume 42.

Holve, R., Protzel, P., Bernasch, J., Naab, K., 1995. Adaptive Fuzzy Control for Driver Assistance in CarFollowing. In Proceedings of the 3rd European Congress on Intelligent Techniques and Soft Computing - EUFIT'95, Aachen, Germany, pp. 11491153.

Persson, M., Botling, F., Hesslow, E., Johansson, R., 1999. Stop\&Go Controller for Adaptive Cruise Control. Control Applications, Proceedings of the 1999 IEEE Conference on, Vol. 2, pp. 1692-1697.

Kato, S., Tsugawa, S., Tokuda, K., Matsui, T., Fujiri, H., 2002. Vehicle Control Algorithms for Cooperative Driving With Automated Vehicles and Intervehicle Communications. IEEE Transactions on Intelligent Transportation Systems, vol. 3, No. 3, pp. 155 - 161.

Naranjo, J. E., González, C., Reviejo, J., García, R., De Pedro, T., 2003. Adaptive Fuzzy Control for Inter.Vehicle gap Keeping. IEEE Transactions on Intelligent Transportation Systems, vol. 4, No. 3.

Marsh. D., 2003. Radar reflects safer highways. EDN Europe. 